\title{
Predicting Planck Scale and Newtonian Constant from a Yang-Mills Gauge Theory: 1- and 2-Loop Estimates
}

\author{
Rodrigo F. Sobreiro ${ }^{1}$ and Anderson A. Tomaz ${ }^{1,2}$ \\ ${ }^{1}$ Universidade Federal Fluminense (UFF), Instituto de Física, Campus da Praia Vermelha, Avenida General Milton Tavares de Souza, \\ s/n, 24210-346 Niterói, RJ, Brazil \\ ${ }^{2}$ Centro Brasileiro de Pesquisas Físicas (CBPF), Rua Dr. Xavier Sigaud 150, Urca, 22290-180 Rio de Janeiro, RJ, Brazil
}

Correspondence should be addressed to Anderson A. Tomaz; tomaz@cbpf.br

Received 7 July 2016; Accepted 8 September 2016

Academic Editor: Shi-Hai Dong

Copyright (c) 2016 R. F. Sobreiro and A. A. Tomaz. This is an open access article distributed under the Creative Commons Attribution License, which permits unrestricted use, distribution, and reproduction in any medium, provided the original work is properly cited. The publication of this article was funded by SCOAP ${ }^{3}$.

Recently, a model for an emergent gravity based on SO(5) Yang-Mills action in Euclidian 4-dimensional spacetime was proposed. In this work we provide some 1- and 2-loop computations and show that the model can accommodate suitable predicting values for the Newtonian constant. Moreover, it is shown that the typical scale of the expected transition between the quantum and the geometrodynamical theory is consistent with Planck scale. We also provide a discussion on the cosmological constant problem.

\section{Introduction}

Quantization of the gravitational field is one of most import problems in physics since the beginning of the 20th century. The long pursuit of a theory of quantum gravity has generated a variety of theoretical proposals to describe the quantum sector of gravity; see, for instance, Loop Quantum Gravity $[1,2]$, Higher Derivatives Quantum Gravity [3, 4], Causal Sets [5], Causal Dynamical Triangulations [6], String Theory $[7,8]$, Asymptotic Safety [9, 10], Emergent Gravities [4, 11], Hořava-Lifshitz Gravity [12], the Nojiri-Odintsov-HoravaLifshitz Instability Free Gravity [13, 14], Topological Gauge Theories [15], and so on. Each one of these theories carries its own set of advantages and disadvantages. On the other hand, gauge theories are relentless in describing the high energy regime of particle physics [16-18]. Hence, one can question if gravity could also be described by a gauge theory in its high energy sector. In fact, since the seminal papers [19-21] about gauge theoretical descriptions of gravity, it is known that gravity can be, at least, dressed as a gauge theory for the local isometries of spacetime. See also [22, 23]. Although consistent with general relativity, these models also have problems with its quantization.

In [24] it was proposed an induced gravity model from a pure Yang-Mills theory based on de Sitter-type groups. In this model, gravity emerges as an effective phenomenon originated by a genuine Yang-Mills action in flat space. The transition between the quantum gauge sector and the classical geometrical sector is mediated by a mass parameter, identified with the Gribov parameter [25-43]. The combination of the running of this parameter and the running of the coupling parameter would provide a good scenario for an InonuWigner contraction [44] of the gauge group, deforming it to a Poincaré-type group. Because the original action is not invariant under the resulting group, the model actually suffers a dynamical symmetry breaking to Lorentz-type groups. At this point, with the help of the mass parameter, the gauge degrees of freedom are identified with geometrical objects, namely, the vielbein and spin connection. At the same time the mass parameter and the coupling parameter are combined to generate the gravity Newtonian constant and an emergent gravity is realized. Moreover, a cosmological 
constant inherent to the model is also generated. See also $[45,46]$ for details.

The aim of the present work is to provide estimates for the emergent parameters of the model above discussed by applying the usual apparatus of quantum field theory (QFT). We concentrate our efforts on 1- and 2-loop computations. In particular, from the explicit expressions of the running coupling and the Gribov parameters, we are able to fit the actual value of Newtonian constant and to obtain a renormalization group cut-off very close to the Planck scale, as expected to be the transition scale from quantum to classical gravity.

The cosmological constant is an essential point, which can be related to the accelerated expansion of the Universe. Observational data and quantum field theory prediction for the cosmological constant strongly disagree in numerical values [18, 47]. Following [48, 49], we can expect that the cosmological parameter generated by the model should combine with the value found in theoretical calculations by quantum field theory in a way that the effective final value fits the observational data. It is worth mentioning that the cosmological parameter generated by the model is related to the Gribov parameter [24]. In [50], a preliminary estimative for these running parameters at 1-loop approximation was scratched. From this reasonable starting, we develop here a refinement on early predictions and we show a numerical improvement at 1-loop calculations. Further, we improve the techniques up to 2-loop estimates. Hence, we present a best estimative for the Gribov parameter in order to fit the model with a suitable emergent gravity.

This work is organized as follows. In Section 2 we resume some concepts and ideas about our effective gravity model. In Section 3, we present the first results that we obtained for 1-loop estimates. In Section 4, we present the main calculations results for running parameters at 2 loops are performed. In Section 5, we discuss shortly our results and some perspectives will be cast.

\section{Effective Gravity from a Gauge Theory}

In [24], a quantum gravity theory was constructed based on an analogy with quantum chromodynamics; see also [45, 46]. In this section we will briefly discuss the main ideas, definitions, and conventions behind this model ${ }^{1}$.

The starting action is the Yang-Mills action:

$$
S_{\mathrm{YM}}=\frac{1}{2} \int F_{A}{ }^{B} * F_{B}{ }^{A}
$$

where $F^{A}{ }_{B}$ are the field strength 2-form and $F=d Y+$ $\kappa Y Y$, where $d$ is the exterior derivative, $\kappa$ is the coupling parameter, and $Y$ is the gauge connection 1-form, that is, the fundamental field in the adjoint representation. The Hodge dual operator in 4-dimensional Euclidian spacetime is denoted by $*$. The action equation (1) is invariant under $S O(5)$ gauge transformations, $Y \mapsto U^{-1}(1 / \kappa d+Y) U$, with $U \in$ $S O(5)$. The infinitesimal version of the gauge transformation is

$$
Y \longmapsto Y+\nabla \alpha
$$

where $\nabla=d+\kappa Y$ is the full covariant derivative and $\alpha$ is the infinitesimal gauge parameter.

It is possible to decompose the gauge group according to $S O(5)=S O(4) \otimes S(4)$, where $S O(4)$ is the stability group $S(4)$ is the symmetric coset space. Thus, defining $J^{5 a} \equiv J^{a}$, the gauge field is also decomposed:

$$
Y=Y_{B}^{A} J_{A}{ }^{B}=A^{a}{ }_{b} J_{a}^{b}+\theta^{a} J_{a},
$$

where capital Latin indices $A, B, \ldots$ run as $\{5,0,1,2,3\}$ and the small Latin indices $a, b, \ldots$ vary as $\{0,1,2,3\}$. The decomposed field strength reads

$$
F=F_{B}^{A} J_{A}{ }^{B}=\left(\Omega^{a}{ }_{b}-\frac{\kappa}{4} \theta^{a} \theta_{b}\right) J_{a}^{b}+K^{a} J_{a},
$$

where $\Omega^{a}{ }_{b}=d A^{a}{ }_{b}+\kappa A^{a}{ }_{c} A^{c}{ }_{b}$ and $K^{a}=d \theta^{a}+\kappa A^{a}{ }_{b} \theta^{b}$. Thus, it is a simple task to find that the Yang-Mills action equation (1) can be rewritten as

$$
\begin{aligned}
S_{\mathrm{YM}} & =\frac{1}{2} \int\left\{\Omega^{a}{ }_{b} * \Omega_{a}{ }^{b}+\frac{1}{2} K^{a} * K_{a}-\frac{\kappa}{2} \Omega^{a}{ }_{b} *\left(\theta_{a} \theta^{b}\right)\right. \\
& \left.+\frac{\kappa^{2}}{16} \theta^{a} \theta_{b} *\left(\theta_{a} \theta^{b}\right)\right\} .
\end{aligned}
$$

Before we advance to the next stage of the model, let us quickly point out some important aspects of YangMills theories and their analogy with a possible quantum gravity model. To start with, Yang-Mills theories present two very important properties, namely, renormalizability and asymptotic freedom [16]. The Yang-Mills action is, in fact, renormalizable, at least to all orders in perturbation theory [51] which means that it is stable at quantum level. In this context, the so-called BRST symmetry has a fundamental hole. Asymptotic freedom $[52,53]$, on the other hand, means that, at high energies, the coupling parameter is very small and we can use perturbation theory in our favor. However, as the energy decreases, the coupling parameter increases and the theory becomes highly nonperturbative. In this regime, the so-called Gribov ambiguities problem takes place [25, 26]. Essentially, the gauge fixing ${ }^{2}$ is not strong enough to eliminate all spurious degrees of freedom from the FaddeevPopov path integral; a residual gauge symmetry survives the Faddeev-Popov procedure. The elimination of the Gribov ambiguities is not entirely understood; however, it is known that a mass parameter is required and a soft BRST symmetry breaking associated with this parameter appears; see, for instance, [30-32, 34, 37, 54, 55]. This parameter is known as Gribov parameter $\gamma$, and it is fixed through minimization of the quantum action, $\delta \Sigma / \delta \gamma^{2}=0$, the so-called gap equation. The action that describes the improved theory (free of infinitesimal ambiguities) is known as Gribov-Zwanziger action [54] and has a more refined version [27, 34] by taking into account few-dimension two operators and their condensation effects.

It is clear that the field $\theta$ has the same degrees of freedom that a soldering forms in spacetime manifold (the vielbein). However, the field $\theta$ carries UV dimension 1 while the vielbein 
is dimensionless. The presence of a mass scale is then very important to identify the field $\theta$ with an effective soldering form. We will show in the next sections that the Gribov parameter is a very good candidate for this purpose. The next step is to perform the rescalings

$$
\begin{aligned}
& A \longrightarrow \frac{1}{\kappa} A, \\
& \theta \longrightarrow \frac{\gamma}{\kappa} \theta,
\end{aligned}
$$

at the action equation (5), achieving

$$
\begin{aligned}
S= & \frac{1}{2 \kappa^{2}} \int\left[\bar{\Omega}_{b}^{a} * \bar{\Omega}_{a}^{b}+\frac{\gamma^{2}}{2} \bar{K}^{a} * \bar{K}_{a}-\frac{\gamma^{2}}{2} \bar{\Omega}_{b}^{a}\right. \\
& \left.*\left(\theta_{a} \theta^{b}\right)+\frac{\gamma^{4}}{16} \theta^{a} \theta_{b} *\left(\theta_{a} \theta^{b}\right)\right],
\end{aligned}
$$

where $\bar{\Omega}_{b}^{a}=d A^{a}{ }_{b}+A^{a}{ }_{c} A^{c}{ }_{b}, \bar{K}^{a}=D \theta^{a}$, and the covariant derivative is now $D=d+A$.

The transition from the action (7) to a gravity action is performed by studying the running behaviour of the quantity $\gamma / \kappa$. It is expected [24] that this quantity vanishes for a specific energy scale. This property induces an Inonu-Wigner contraction $S O(5) \mapsto I S O(4)$ [44]. However, since the action (7) is not invariant under ISO(4) gauge transformations, the theory actually suffers a symmetry breaking to the stability group $S O(4)$. The broken theory is ready to be rewritten as a gravity theory. The map (see [24]) is a simple identification of the gauge fields with geometric effective entities according to $\delta_{\mathfrak{a}}^{a} \delta_{b}^{\mathfrak{b}} A_{a}{ }^{b}=\omega_{\mathfrak{a}}^{\mathfrak{b}}$ and $\delta_{a}^{\mathfrak{a}} \theta^{a}=e^{\mathfrak{a}}$. Where the indices $\{\mathfrak{a}, \mathfrak{b}$, $c, \ldots\}$ belong to the tangent space of the effective deformed spacetime, $\omega$ is the spin connection 1-form and $e$ is the vielbein 1-form. Thus, with the extra parametric identifications

$$
\gamma^{2}=\frac{\kappa^{2}}{4 \pi G}=\frac{4 \Lambda^{2}}{3}
$$

where $G$ is the Newtonian constant and $\Lambda^{2}$ is the renormalized cosmological constant ${ }^{3}$. Hence, the action equation (7) generates the following effective gravity action:

$$
\begin{aligned}
& S_{\text {Grav }}=\frac{1}{16 \pi G} \int\left\{\frac{3}{2 \Lambda^{2}} R_{\mathfrak{a}}^{\mathfrak{b}} \star R^{\mathfrak{a}}{ }_{\mathfrak{b}}-\frac{1}{2} \epsilon_{\mathfrak{a} \mathfrak{b} \mathfrak{d} \mathfrak{d}} R^{\mathfrak{a} \mathfrak{b}} e^{\mathfrak{c}} e^{\mathfrak{d}}+T^{\mathfrak{a}}\right. \\
& \left.\star T_{\mathfrak{a}}+\frac{\Lambda^{2}}{12} \epsilon_{\mathfrak{a} \mathfrak{b} \mathfrak{d} \mathfrak{d}} e^{\mathfrak{a}} e^{\mathfrak{b}} e^{\mathfrak{c}} e^{\mathfrak{d}}\right\},
\end{aligned}
$$

where $R^{\mathfrak{a}}{ }_{\mathfrak{b}}=d \omega^{\mathfrak{a}}{ }_{\mathfrak{b}}+\omega^{\mathfrak{a}}{ }_{\mathfrak{c}} \omega^{\mathfrak{c}}{ }_{\mathfrak{b}}$ and $T^{\mathfrak{a}}=d e^{\mathfrak{a}}+\omega^{\mathfrak{a}}{ }_{\mathfrak{b}} e^{\mathfrak{b}}$ are, respectively, the curvature and torsion 2-forms. The symbol * stands for the Hodge dual operator in $\mathbb{M}^{4}$ (the deformed spacetime).

\section{Running Parameters and 1-Loop Estimates}

Now $^{4}$, we return to the original action (1) and we take only its quadratic part ${ }^{5}$, considering also the Gribov-Zwanziger quadratic term ${ }^{6}$ [27],

$$
\begin{aligned}
S_{\text {quad }} & =\int d^{4} x\left\{\frac{1}{4}\left(\partial_{\mu} Y_{\nu}^{A}-\partial_{\nu} Y_{\mu}^{A}\right)^{2}+\frac{1}{2 \alpha}\left(\partial_{\mu} Y_{\mu}^{A}\right)^{2}\right. \\
+ & \bar{\varphi}_{\mu}^{A B} \partial^{2} \varphi_{\mu}^{A B}+-\lambda^{2} \kappa\left(f^{A B C} Y_{\mu}^{A} \varphi_{\mu}^{B C}+f^{A B C} Y_{\mu}^{A} \bar{\varphi}_{\mu}^{B C}\right) \\
& \left.-\lambda^{4} d\left[\frac{N(N-1)}{2}\right]\right\},
\end{aligned}
$$

where $\left(\varphi_{\mu}^{A B}, \bar{\varphi}_{\mu}^{A B}\right)$ is a pair of complex conjugate bosonic fields and $\lambda$ is, essentially, the Gribov parameter. Here we will use $N=5$ since we are building a Yang-Mills for the $S O(5)$ group. In the future we will employ this value, but, for now, we continue using $N$ in general. The parameter $\alpha$ is the gauge parameter associated with the gauge fixing. Herein, the limit $\alpha \rightarrow 0$ must be employed in order to enforce the Landau gauge condition; that is, $\partial_{\mu} Y_{\mu}^{A}=0$. The choice of the gauge is, in principle, arbitrary as long as the gauge is renormalizable. However, most of the developments in the Gribov problem were made in the Landau gauge $[25,27,30$, 34]. Moreover, the Landau gauge is a very simple gauge to work with. Nevertheless, there are recent evidences that the Gribov parameter is a gauge invariant parameter [40, 41, 43, $56,57]$, a very welcome feature for the model.

At 1-loop, the effective action ${ }^{7}$ is defined through

$$
e^{-\Gamma^{(1)}}=\int[D \Phi] e^{-S_{\text {quad }}}
$$

which, in $d$ dimensions, yields

$$
\begin{gathered}
\Gamma^{(1)}=-\lambda^{4} d\left[\frac{N(N-1)}{2}\right]+\frac{(d-1)}{2}\left[\frac{N(N-1)}{2}\right] \\
\cdot \int \frac{d^{d} p}{(2 \pi)^{d}}\left[\ln \left(p^{4}+2 N \kappa^{2} \lambda^{4}\right)\right] .
\end{gathered}
$$

To control the divergences of the quantum action we employ the $\overline{\mathrm{MS}}$ renormalization scheme to obtain

$$
\begin{gathered}
\Gamma_{r}^{(1)}=-\lambda^{4} d\left[\frac{N(N-1)}{2}\right]-\frac{(d-1)}{32 \pi^{2}}\left[\frac{N(N-1)}{2}\right] \\
\cdot\left(N \kappa^{2} \lambda^{4}\right)\left[\ln \left(\frac{2 N \kappa^{2} \lambda^{4}}{\mu^{4}}\right)-\frac{8}{3}\right],
\end{gathered}
$$

where

$$
\gamma^{4} \equiv 2 \kappa^{2} \lambda^{4}
$$

is a more convenient mass parameter. At first sight, this choice is a mere algebraic ansatz to simplify future computations with this parameter. In Appendix B we demonstrate why this 
choice is actually better than $\lambda$ for our purposes. Thus, for $d=4$, (13) turns to

$$
\begin{aligned}
\Gamma_{r}^{(1)}= & -\frac{\gamma^{4}}{2 k^{2}} 4\left[\frac{N(N-1)}{2}\right] \\
& -\frac{3}{32 \pi^{2}}\left[\frac{N(N-1)}{2}\right] \frac{N \gamma^{4}}{2}\left[\ln \left(\frac{N \gamma^{4}}{\mu^{4}}\right)-\frac{8}{3}\right] .
\end{aligned}
$$

Following the Gribov-Zwanziger prescription [28], the Gribov parameter can be determined by minimizing the quantum action; that is, $\partial \Gamma_{r}^{(1)} / \partial \gamma^{2}=0$. The result is

$$
\frac{N \kappa^{2}}{16 \pi^{2}}\left[\frac{5}{8}-\frac{3}{8} \ln \left(\frac{N \gamma^{4}}{\mu^{4}}\right)\right]=1 .
$$

Or, equivalently,

$$
\gamma^{2}=\frac{e^{5 / 6}}{\sqrt{N}} \mu^{2} e^{-(4 / 3)\left(16 \pi^{2} / N \kappa^{2}\right)} .
$$

Moreover, the 1-loop coupling parameter is found to be [52]

$$
\frac{N \kappa^{2}}{16 \pi^{2}}=\frac{1}{(11 / 3) \ln \left(\mu^{2} / \bar{\Lambda}^{2}\right)},
$$

where $\bar{\Lambda}$ is the renormalization group cut-off. By inserting (18) into (17) for $N=5$ we find

$$
\gamma^{2}=\frac{e^{5 / 6}}{\sqrt{5}} \bar{\Lambda}^{2}\left(\frac{\mu^{2}}{\bar{\Lambda}^{2}}\right)^{-35 / 9} .
$$

Thus, the higher the energy scale is, the smaller the Gribov parameter would be. This behaviour is plotted in Figure 1.

As we have mentioned in Section 2, the ratio between the two quantum parameters, after we combine (18) and (19), namely,

$$
\frac{\gamma^{2}}{\kappa^{2}}=\alpha \bar{\Lambda}^{2}\left(\frac{\mu^{2}}{\bar{\Lambda}^{2}}\right)^{-35 / 9} \ln \left(\frac{\mu^{2}}{\bar{\Lambda}^{2}}\right),
$$

with $\alpha=55 e^{5 / 6} /\left(48 \pi^{2} \sqrt{5}\right)$, is crucial for the present model. The behaviour of this ratio is illustrated in Figure 2. It is clear that the expected behaviour $\gamma^{2} / \kappa^{2} \rightarrow 0$ is attained at $\mu^{2}=\bar{\Lambda}^{2}$.

The simple inversion of (20) gives

$$
\frac{\kappa^{2}}{\gamma^{2}}=\frac{1}{\alpha \bar{\Lambda}^{2}}\left(\frac{\mu^{2}}{\bar{\Lambda}^{2}}\right)^{35 / 9}\left[\frac{1}{\ln \left(\mu^{2} / \bar{\Lambda}^{2}\right)}\right],
$$

which shows the running behaviour of the ratio $\kappa^{2} / \gamma^{2}$ which is displayed in Figure 3. We remark that there is a discontinuity (Landau pole) at $\mu=\bar{\Lambda}$. We interpret this discontinuity as an indication of the transition between the quantum and classical regimes of the model. For $\mu<\bar{\Lambda}$ we expect a geometrical regime while for $\mu>\bar{\Lambda}$, the theory is at the quantum region.

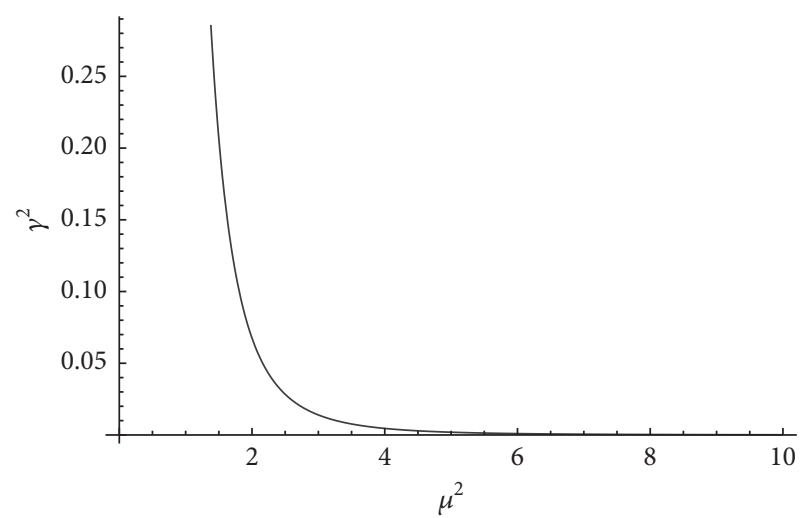

FIGURE 1: The running of the Gribov parameter as function of the energy scale squared. The energy squared $\mu^{2}$ is in units of $\bar{\Lambda}^{2}$ and the Gribov parameter is normalized in units of $\left(e^{5 / 6} / \sqrt{5}\right) \bar{\Lambda}^{2}$.

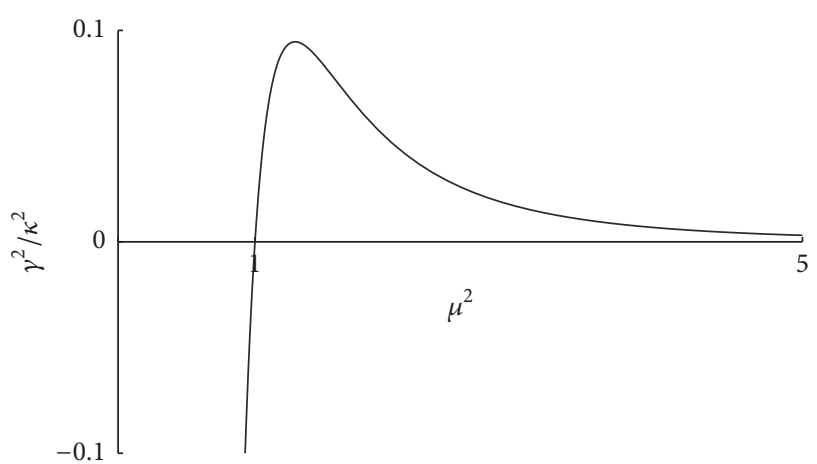

Figure 2: The running of the ratio $\gamma^{2} / \kappa^{2}$ as function of energy scale squared. The energy scale $\mu^{2}$ is in units of $\bar{\Lambda}^{2}$ and the ratio is in units of $\alpha \bar{\Lambda}^{2}$.

To estimate the Newtonian constant and the renormalization group cut-off we emphasize that we are not assigning any running behaviour to the Newtonian constant. According to (8), our aim consists in identifying the ratio $\gamma^{2} / \kappa^{2}$ with $G$ only after an energy scale is chosen. So the Newtonian constant is fixed as an effective quantity.

It is also important to realize that the deep infrared behaviour of Figures 1, 2, and 3 does not reproduce the expected behaviour at zero momenta, as known by QCD lattice simulations [58-63]. In the deep infrared regime the coupling parameter $\kappa$ goes to a finite value, that is, an infrared fixed point. However, this extreme behaviour is not relevant for the purposes of the present work.

3.1. Numerical Estimates at 1-Loop. We first follow the procedure performed in [50] where the strategy was not to solve the gap equation by fixing $\bar{\Lambda}$ and $\mu$, which is the traditional way, but to fix the Newtonian constant and find if this is a consistent solution. Nevertheless, for the sake of consistency, we need a coupling constant as small as possible. 


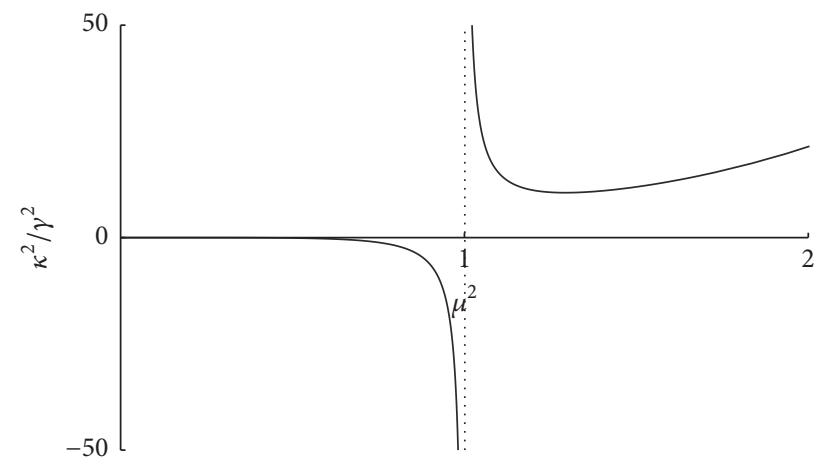

FIGURE 3: The ratio $\kappa^{2} / \gamma^{2}$ as a function of the energy scale squared. The energy scale is in units of $\bar{\Lambda}^{2}$ and the ratio is in units of $1 /\left(\alpha \bar{\Lambda}^{2}\right)$.

Furthermore, we must have $\mu^{2}>\bar{\Lambda}^{2}$. Accordingly, we possess a certain range to work with; namely,

$$
\begin{aligned}
& 0<\frac{N \kappa^{2}}{16 \pi^{2}}<1, \\
& 0<\ln \left(\frac{\mu^{2}}{\bar{\Lambda}^{2}}\right)<1 .
\end{aligned}
$$

Let us take, for instance, $\mu^{2}=2 \bar{\Lambda}^{2}$ in (21), which provides $\ln \left(\mu^{2} / \bar{\Lambda}^{2}\right)=0.6931$, satisfying Ineq. (22). One way to obtain the scale $\bar{\Lambda}$ is setting the Newtonian constant to its experimental value; that is, $G=6.707 \times 10^{-33} \mathrm{TeV}^{-2}$ in (21), providing

$$
\bar{\Lambda}^{2} \approx 2.122 \times 10^{33} \mathrm{TeV}^{2}
$$

This result allows us to estimate the renormalized cosmological constant. Combining (8), (19), and (23) we obtain

$$
\Lambda^{2} \approx 1.106 \times 10^{32} \mathrm{TeV}^{2} \text {. }
$$

We notice that the cut-off value (23) is just right above the Planck scale, given by $E_{p}^{2}=1.491 \times 10^{32} \mathrm{TeV}^{2}$.

3.1.1. Methods of Enhancement at 1 Loop. The main goal here is to calculate the best values for $N \kappa^{2} / 16 \pi^{2}$ and $\ln \left(\mu^{2} / \bar{\Lambda}^{2}\right)$ in accordance with Ineq. (22). To handle this task we apply three methods, labelled by $M_{1}, M_{2}$, and $M_{3}$, as follows.

$M_{1}$ : Taylor Series Method. Let us rewrite (18) as

$$
\frac{1}{a}=\frac{11}{3} \ln b
$$

where

$$
\begin{aligned}
& a=\frac{N \kappa^{2}}{16 \pi^{2}}, \\
& b=\frac{\mu^{2}}{\bar{\Lambda}^{2}},
\end{aligned}
$$

for mere simplification. Next, we expand the right hand side of (25) as a Taylor series at the critical point $\mu=\bar{\Lambda}$, that is, $b=1$, as follows:

$$
\ln (b)=\sum_{n=1}^{\infty} \frac{1}{n}(-1)^{n-1}(b-1)^{n},
$$

with $0<\ln b<1$ as stated by Ineq. (22). We investigate the series (27) under two perspectives as follows.

Perspective (i): The Endpoint Extremum. The series expansion of $\ln (b)$ has radius of convergence equal to 1 . Precisely, the alternating series test ensures that the series does not converge at $b=0$ and converges at $b=2$. Hence, the series is convergent for $0<b \leqslant 2$. Therefore, the endpoint extremum occurs at $b=2$ which happens also to be a global maximum. A curious fact about that series occurs when we truncate the series expansion at even $n$th order: any of these truncations have a maximum at $b=2$. Again such maximum is a global one and it happens at the endpoint. From (25), it is clear that, by fixing a global maximum for $\ln (b)$, a minimum value for $N \kappa^{2} / 16 \pi^{2}$ is set. Thus, with $b=2$, we obtain $\ln \left(\mu^{2} / \bar{\Lambda}^{2}\right)=0.6931$ and $N \kappa^{2} / 16 \pi^{2}=0.3935$, which are both in accordance with the intervals described in Ineq. (22).

Perspective (ii): A Bound on the Taylor Series for $\ln (b)$. At this point, we are looking for a certain bound for the series expansion (27). Hence, since $a<1$, we have

$$
\begin{aligned}
\ln (b) & >\frac{3}{11} \Longleftrightarrow \\
\sum_{n=1}^{\infty} \frac{1}{n}(-1)^{n-1}(b-1)^{n} & >\frac{3}{11} .
\end{aligned}
$$

In this range we solve the Ineq. (28) for several $n$ values. We notice that the choices of $b$ values are restricted to $1.314<b<$ $b_{\text {sup }}$ while $n$ is even and where $b_{\text {sup }}$ values decrease while even $n$ values increase. The $b_{\text {sup }}$ values are displayed in Table 1 .

Still looking at Table 1 , for instance, $n=8 \Rightarrow b_{\text {sup }} \approx 2.305$, $n=10 \Rightarrow b_{\text {sup }} \approx 2.261$, and-as actually we would expect$n \rightarrow \infty \Rightarrow b_{\text {sup }} \rightarrow 2.000$. However, if $n$ is odd we obtain for all intervals $b>1.313$, of course, and no upper bound. Hence, we have a confirmation of our first choice for $\ln \left(\mu^{2} / \bar{\Lambda}^{2}\right)$ given by $\mu^{2}=2 \bar{\Lambda}^{2}$.

Besides the best choice that we can perform, we still have freedom to choose any value consistent with Ineq. (22). If we pick, for instance, $a=0.4300$, we have $b=1.886 \Rightarrow \ln (b) \equiv$ $\ln \left(\mu^{2} \bar{\Lambda}^{2}\right) \approx 0.6342$, which provides, from (21) and (8),

$$
\begin{aligned}
& \bar{\Lambda}^{2} \approx 1.845 \times 10^{33} \mathrm{TeV}^{2}, \\
& \Lambda^{2} \approx 1.208 \times 10^{32} \mathrm{TeV}^{2} .
\end{aligned}
$$

These results can be interpreted as a numerical verification of the values equations (23) and (24) due to the fact that their order of magnitude are maintained. In this sense we confirm the first insight presented in [50]. 
TABLE 1: The superior bound for $b$ range using only even values for $n$.

\begin{tabular}{lc}
\hline$n$ & $b_{\text {sup }}$ \\
\hline 2 & 2.674 \\
4 & 2.476 \\
8 & 2.305 \\
10 & 2.261 \\
20 & 2.158 \\
50 & 2.079 \\
100 & 2.046 \\
1000 & 2.007 \\
5000 & 2.002 \\
10000 & 2.000 \\
\hline
\end{tabular}

$M_{2}$ : Equilibrium Value Method. Now we use a simple method of enhancement which we called equilibrium value between two functions at certain point. First, in order to simplify, we take (18) as

$$
f\left(\kappa^{2}\right) h\left(\mu^{2}, \bar{\Lambda}^{2}\right)=\frac{3}{11}
$$

where

$$
\begin{gathered}
f\left(\kappa^{2}\right)=\frac{N \kappa^{2}}{16 \pi^{2}}, \\
h\left(\mu^{2}, \bar{\Lambda}^{2}\right)=\ln \left(\frac{\mu^{2}}{\bar{\Lambda}^{2}}\right) .
\end{gathered}
$$

To obtain small values for $h\left(\mu^{2}, \bar{\Lambda}^{2}\right)$ and $f\left(\kappa^{2}\right)$, we made an equilibrium choice; that is, $h\left(\mu^{2}, \bar{\Lambda}^{2}\right)=f\left(\kappa^{2}\right)$. Consequently, it provides

$$
\begin{aligned}
h\left(\mu^{2}, \bar{\Lambda}^{2}\right) & =\left(\frac{3}{11}\right)^{1 / 2} \Longrightarrow \\
\ln \left(\frac{\mu^{2}}{\bar{\Lambda}^{2}}\right) & \approx 0.5222 \Longrightarrow \\
\frac{\mu^{2}}{\bar{\Lambda}^{2}} & \approx 1.686 .
\end{aligned}
$$

Thus, from (21) and (8), we find

$$
\begin{aligned}
& \bar{\Lambda}^{2} \approx 1.449 \times 10^{33} \mathrm{TeV}^{2}, \\
& \Lambda^{2} \approx 1.468 \times 10^{32} \mathrm{TeV}^{2} .
\end{aligned}
$$

We conclude that (34) and (35) do not show any significant improvement with respect to (23) and (24).

$M_{3}$ : Method by Geometrical Series. Here we employ a geometrical series to treat the logarithm that will be used. Due to Ineq. (22), we can treat the logarithm in (18) as a geometrical series. First, we define

$$
r=1-\ln \frac{\mu^{2}}{\bar{\Lambda}^{2}} .
$$

TABLE 2: The superior bound $r_{\text {sup }}$ for the range of values for $r$ as a function of the of $n$ th-degree polynomial.

\begin{tabular}{lc}
\hline$n$ & $r_{\text {sup }}$ \\
\hline 5 & 0.7974 \\
8 & 0.7470 \\
10 & 0.7367 \\
20 & 0.7276 \\
30 & 0.7273 \\
40 & 0.7273 \\
100 & 0.7273 \\
1000 & 0.7273 \\
\hline
\end{tabular}

Hence, we can use ${ }^{8}$

$$
\frac{1}{1-r}=\sum_{n=0}^{\infty} r^{n}
$$

in (18), providing

$$
\frac{N \kappa^{2}}{16 \pi^{2}}=\frac{3}{11}\left(\frac{1}{1-r}\right)=\frac{3}{11} \sum_{n=0}^{\infty} r^{n} .
$$

Second, we use Ineq. (22) and (38) to write

$$
\sum_{n=0}^{\infty} r^{n}<\frac{11}{3}
$$

Now, we test several truncations of expression equation (39) to deal with an $n$ th-degree polynomial inequality. Such procedure permits us to find $r \in(0,0.7273)$ as an optimum valid range. To clarify this point, for instance, we mount Table 2 displaying the evolution of this range, which directly determines the value of the logarithm.

We notice that $n>30$ does not bring any significant improvement for the superior bound of $r$. In this way, we choose $r \approx 0.7273$ as an optimal extreme valid value, which implies that $\ln \left(\mu^{2} / \bar{\Lambda}^{2}\right) \approx 0.2727$ and $N \kappa^{2} / 16 \pi^{2} \approx 0.3803$. With these values and using (21) and (8) we find the following results:

$$
\begin{aligned}
& \bar{\Lambda}^{2} \approx 1.052 \times 10^{32} \mathrm{TeV}^{2}, \\
& \Lambda^{2} \approx 2.810 \times 10^{32} \mathrm{TeV}^{2} .
\end{aligned}
$$

Then, $\bar{\Lambda}^{2}$ decreases by one order of magnitude when compared to (23), (29), and (34). It is straightforward to see how these values can be obtained directly from (38). We stress out that the superior bound for $n<30$ leaves us with an invalid range for $N \kappa^{2} / 16 \pi^{2}$.

For the other extreme we choose $r=1.000 \times 10^{-4}$, providing $\ln \left(\mu^{2} / \bar{\Lambda}^{2}\right) \approx 0.9999$ and $N \kappa^{2} / 16 \pi^{2} \approx 0.2728$. We use these values to find

$$
\begin{aligned}
& \bar{\Lambda}^{2} \approx 4.851 \times 10^{33} \mathrm{TeV}^{2}, \\
& \Lambda^{2} \approx 7.666 \times 10^{31} \mathrm{TeV}^{2} .
\end{aligned}
$$


TABLE 3: The cut-off and renormalized cosmological constant obtained in each method. The column $I$ lists our initial estimates. The other columns $M_{1}, M_{2}, M_{3 a}$, and $M_{3 b}$ are related to the values obtained by Taylor series, equilibrium value, and geometric series, respectively. The column Pr exhibits the physical predictions, that is, the Planck energy squared and the absolute value predicted by the quantum field theory for the cosmological constant [47].

\begin{tabular}{|c|c|c|c|c|c|c|}
\hline & $I$ & $M_{1}$ & $M_{2}$ & $M_{3 a}$ & $M_{3 b}$ & $\operatorname{Pr}$ \\
\hline $\bar{\Lambda}^{2}\left(\mathrm{TeV}^{2}\right)$ & $2.122 \times 10^{33}$ & $1.845 \times 10^{33}$ & $1.449 \times 10^{33}$ & $1.052 \times 10^{32}$ & $4.851 \times 10^{33}$ & $1.491 \times 10^{32}$ \\
\hline$\Lambda^{2}\left(\mathrm{TeV}^{2}\right)$ & $1.106 \times 10^{32}$ & $1.208 \times 10^{32}$ & $1.468 \times 10^{32}$ & $2.810 \times 10^{32}$ & $7.666 \times 10^{31}$ & $3.710 \times 10^{28}$ \\
\hline
\end{tabular}

TABLE 4: Newtonian constant and cosmological constant values based on the logarithm computed in each method in Section 3.1 with the cut-off equal to the Planck energy.

\begin{tabular}{|c|c|c|c|c|c|}
\hline & $I$ & $M_{1}$ & $M_{2}$ & $M_{3 a}$ & $M_{3 b}$ \\
\hline$G_{p}\left(\mathrm{TeV}^{-2}\right)$ & $9.551 \times 10^{-32}$ & $8.301 \times 10^{-32}$ & $6.521 \times 10^{-32}$ & $5.254 \times 10^{-32}$ & $2.183 \times 10^{-31}$ \\
\hline$\Lambda_{p}(\mathrm{TeV})$ & $7.766 \times 10^{30}$ & $9.765 \times 10^{30}$ & $1.510 \times 10^{31}$ & $6.271 \times 10^{31}$ & $2.355 \times 10^{30}$ \\
\hline
\end{tabular}

In this case a better value for the renormalized cosmological constant is found. However, the renormalization group cutoff is the worst found until this point. To summarize all results that we found in each method we built Table 3.

Comparing the numerical values for the cut-off and the renormalized cosmological constant which were obtained through the three methods $M_{1}, M_{2}$, and $M_{3}$ and listed in Table 3, we observe that the orders of magnitude of those results are almost unchanged. A unique exception occurs to the cut-off in the column $M_{3 b}$, which is caused by the extreme high value to the logarithm $\ln \left(\mu^{2} / \bar{\Lambda}^{2}\right)$.

3.1.2. Fixing $\bar{\Lambda}$ as the Planck Energy. We introduce here a different path to find values for the Newtonian constant and the renormalized cosmological constant. In this manner we made all slightly different since we fit the cut-off $\bar{\Lambda}^{2}$ equal to energy Planck; that is, $\bar{\Lambda}^{2}=E_{p}^{2}=1.491 \times 10^{16} \mathrm{TeV}$. Previously, in Section 3.1, we found an optimum logarithm to fix the cut-off and the renormalized cosmological constant. With the help of fixed logarithms and (21) we compute the Newtonian constant $G_{p}$ for each method as displayed in Table 4 .

We observe that all values for $G_{p}$ are in 1 order of magnitude above $G$. After confrontation with the values presented in Table 2 we notice a better estimate using method $M_{3 b}$, that is, while we apply the logarithm obtained with the geometrical series for $\kappa^{2}$. The method $M_{3 a}$ ensures the best value for $G=6.707 \times 10^{-33} \mathrm{TeV}^{2}$. As a consequence, the price to pay is a renormalized cosmological constant with a higher value than one encountered in the method $M_{3 b}$. However, the order of magnitude is the same while we compare the values for $\Lambda^{2}$ found through the methods $M_{3 a}$ and $M_{3 b}$.

\section{Numerical Estimates at 2 Loops}

For 2 loops, an explicit analytical computation is a virtually impossible task. To work out this equation, sophisticated algebraic programs were built. For instance, FORM and QGraph programs are frequently used as well as the recent developments about such computational packages [64-69].

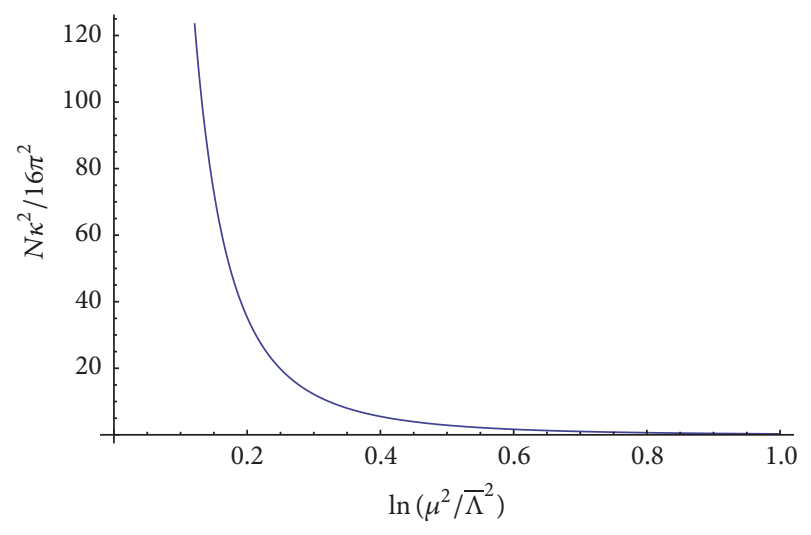

FIGURE 4: The behaviour of the coupling parameter related to the energy scale.

In this section we borrow the main results of 2-loop computations from [65].

4.1. 2-Loop $\beta$-Function. First, recalling that the $\beta$-function at 2 loops $[65,70]$ is given by

$$
\beta\left(\kappa^{2}\right)=-\frac{11 N}{3}\left(\frac{\kappa^{2}}{16 \pi^{2}}\right)^{2}-\frac{34}{3} N^{2}\left(\frac{\kappa^{2}}{16 \pi^{2}}\right)^{3},
$$

the 2-loop running coupling constant is

$$
\begin{aligned}
\frac{N \kappa^{2}}{16 \pi^{2}}= & \frac{1}{(11 / 3) \ln \left(\mu^{2} / \bar{\Lambda}^{2}\right)} \\
& -\frac{102}{121}\left\{\frac{\ln \left[\ln \left(\mu^{2} / \bar{\Lambda}^{2}\right)\right]}{\left[\ln \left(\mu^{2} / \bar{\Lambda}^{2}\right)\right]^{2}}\right\},
\end{aligned}
$$

where $\bar{\Lambda}$ is the cut-off of the energy scale. The evolution of the coupling $\kappa$, related to energy scale $\mu$, is displayed in Figure 4.

4.2. 2-Loop Gap Equation. From [65], the main result is the 2-loop Gribov gap equation in the $\overline{\mathrm{MS}}$ with massive quarks. 
Here, we are dealing with a theory without fermions. Hence, from [65], the 2-loop gap equation reduces to the simpler form:

$$
\begin{aligned}
1= & \left(\frac{N \kappa^{2}}{16 \pi^{2}}\right)\left[\frac{5}{8}-\frac{3}{8} \ln \left(\frac{N \gamma^{4}}{\mu^{4}}\right)\right]+\left(\frac{N \kappa^{2}}{16 \pi^{2}}\right)^{2} \\
& \cdot\left\{\frac{3893}{1536}+\frac{825}{4096} \sqrt{3} \pi^{2}+\frac{29}{768} \pi^{2}-\frac{65}{48} \ln \left(\frac{N \gamma^{4}}{\mu^{4}}\right)\right. \\
& \left.+\frac{35}{128} \ln ^{2}\left(\frac{N \gamma^{4}}{\mu^{4}}\right)+\frac{137}{2048} \sqrt{5} \pi^{2}-\frac{1317}{4096} \pi^{2}\right\} .
\end{aligned}
$$

$$
\begin{aligned}
& h(\mu)=\ln \left(\frac{\mu^{2}}{\bar{\Lambda}^{2}}\right), \\
& \mathscr{H}(\mu)=\frac{1496}{105} \frac{\mathscr{P}(\mu)}{Q}, \\
& \mathscr{P}(\mu)=h(\mu)[33 h(\mu)+65] \text {, } \\
& Q(\mu)=\{11 h(\mu)-34 \ln [h(\mu)]\}^{2}, \\
& \mathscr{W}_{m}(\mu)=\frac{1}{1680 Q(\mu)}[\mathcal{S}(\mu)-\sqrt{2} \mathscr{T}(\mu)] \\
& \mathscr{W}_{p}(\mu)=\frac{1}{1680 Q(\mu)}[\mathcal{S}(\mu)+\sqrt{2} \mathscr{T}(\mu)] \\
& \mathcal{S}(\mu)=a_{1} h^{3}(\mu)+a_{2} h^{2}(\mu)+a_{3} \ln ^{2}[h(\mu)], \\
& \mathscr{T}(\mu)=\sqrt{\mathscr{Q}(\mu)\left\{b_{1} h^{4}(\mu)+b_{2} h^{3}(\mu)-b_{3} h^{2}(\mu)-b_{4} \ln ^{2}[h(\mu)]+b_{5} \ln [h(\mu)] h(\mu)-b_{6} \ln [h(\mu)] h^{2}(\mu)\right\}},
\end{aligned}
$$

First of all, we compute the system formed by (44) and (45) to analyze the behaviour of the Gribov parameter related to energy scale. Such procedure gives us the following two functions, where we have now the mass parameter related to logarithm:

$$
\begin{aligned}
& \gamma_{m}^{2}=\frac{1}{\sqrt{5}} \mu^{2}[h(\mu)]^{-\mathscr{H}(\mu)} e^{\mathscr{W}_{m}(\mu)}, \\
& \gamma_{p}^{2}=\frac{1}{\sqrt{5}} \mu^{2}[h(\mu)]^{-\mathscr{H}(\mu)} e^{\mathscr{W}_{p}(\mu)},
\end{aligned}
$$

where where $a_{1}=255,552, a_{2}=251,680, a_{3}=2,404,480, b_{1}=$ $2,368,796,672, b_{2}=173,775,360, b_{3}=605 d_{0}, b_{4}=5780 d_{0}$, $b_{5}=3740 d_{0}, b_{6}=537,123,840, d_{0}=221,384+b_{0}$, and $b_{0}=21(-3,487+2,475 \sqrt{3}+822 \sqrt{5}) \pi^{2}$.

The behaviour of $\gamma_{m}^{2}$ and $\gamma_{p}^{2}$ in (46) can be clearly seen in Figures 5 and 6 , respectively. The behaviour of $\gamma_{p}^{2}$ and $\gamma_{m}^{2}$ indicates uniquely $\gamma_{m}^{2}$ as the one that has the expected typical running behaviour of a mass parameter in the GribovZwanziger scenario. Thus, we necessarily keep $\gamma_{m}^{2}$ for the next computations.

4.3. Methods of Enhancement at 2 Loops. Following similar steps that we have made in Section 3.1.1, we are looking for the best logarithm for the sake of better estimates of the renormalized cosmological constant $\Lambda^{2}$ and the energy cutoff $\widetilde{\Lambda}^{2}$. Before we advance in applying these methods, we refer to the definitions (32). Moreover, we skip the initial choice, as made at 1 loop, $\mu^{2} / \bar{\Lambda}^{2}=2$, because it provides $N \kappa^{2} / 16 \pi^{2}=1.036$, which is outside the acceptable range for the coupling parameter; see Ineq. (22). Hence, we proceed with the methods of enhancement as we have done in Section 3.1.1.

$M_{1}$ : Taylor Series Method. We are looking for small logarithms through a Taylor expansion of (44), which can be written as

$$
\begin{aligned}
f(\kappa)= & \frac{3}{11}-\frac{135}{121}(h(\mu)-1)+\frac{288}{121}(h(\mu)-1)^{2} \\
& -\frac{475}{121}(h(\mu)-1)^{3}+\frac{125}{22}(h(\mu)-1)^{4} \\
& -\frac{4602}{605}(h(\mu)-1)^{5}+\mathcal{O}\left((h(\mu)-1)^{6}\right),
\end{aligned}
$$

where, for simplicity, the expansion above is displayed up to fifth order. However, we must keep in mind that we can truncate such expansion at any arbitrary order. If we truncate the above expansion of $f(\kappa)$ at fourth order and consider $0<f(\kappa)<1$, then we find $h(\mu)>0.6938$. Therefore, we obtain $0.6938<h(\mu)<1.000$. All truncations beyond the fourth order do not imply significant improvements in the inferior limit $h_{\text {inf }}$, in the interval $h_{\text {inf }}<h(\mu)<1$, of the 


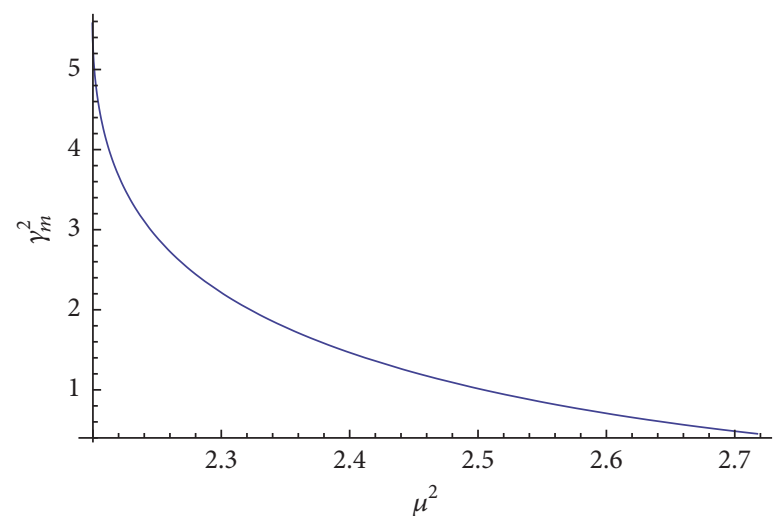

FIGURE 5: Gribov parameter $\gamma_{m}^{2}$ as a function of the energy scale $\mu^{2}$. Both $\gamma_{m}^{2}$ and $\mu^{2}$ are in units of $\bar{\Lambda}^{2}$.

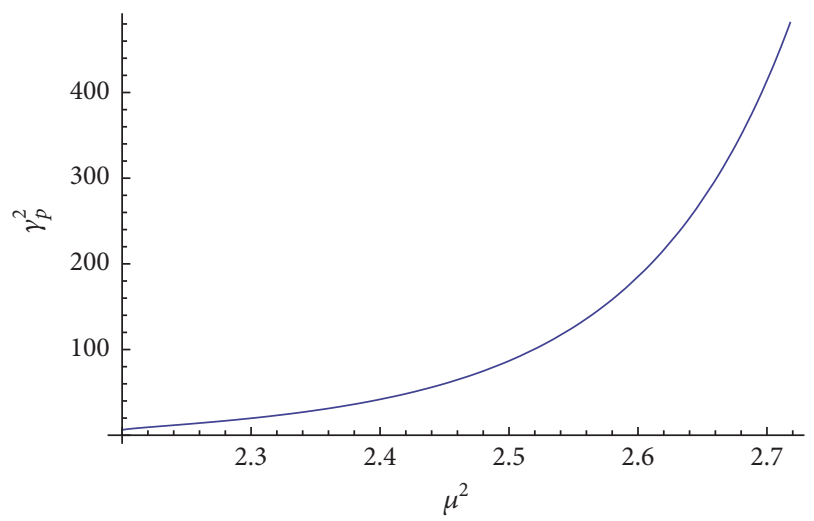

FIGURE 6: Gribov parameter $\gamma_{p}^{2}$ as a function of the energy scale $\mu^{2}$. Both $\gamma_{p}^{2}$ and $\mu^{2}$ are in units of $\bar{\Lambda}^{2}$.

intervals for $h(\mu)$, since each order of truncation modifies such limit (see Table 5).

Since we are dealing with a perturbation expansion, we need to get to small values for $h(\mu)$. Nevertheless, because of the second term in (44), which is resulting from the contribution at 2 loops in the computation, a choice for the logarithm close to any $h_{\text {inf }}$ results in high numerical values of the renormalized cosmological constant $\Lambda^{2}$. Hence, the best choice for the logarithm is $\ln \left(\mu^{2} / \bar{\Lambda}^{2}\right)=0.9999$. Employing this logarithm value and combining (44), (45), and (8), we obtain

$$
\begin{aligned}
& \bar{\Lambda}^{2} \approx 2.269 \times 10^{32} \mathrm{TeV}^{2}, \\
& \Lambda^{2} \approx 7.665 \times 10^{31} \mathrm{TeV}^{2} .
\end{aligned}
$$

The value (49) is very close to the order of magnitude of the Planck energy $E_{p}^{2}$. The result (41) is almost better than that we found in Section 3.1.1 at 1-loop approximation. The result (50) certifies the 1-loop result for $\Lambda^{2}$.
TABLE 5: The inferior limit $h_{\text {inf }}$ for the range of values for $h(\mu)$ according to $n_{t}$, which is the order of truncation of the expansion for $f(\kappa)$.

\begin{tabular}{lc}
\hline$n_{t}$ & $h_{\text {inf }}$ \\
\hline 2 & 0.6340 \\
3 & 0.6806 \\
4 & 0.6938 \\
5 & 0.6983 \\
6 & 0.6998 \\
7 & 0.7004 \\
8 & 0.7006 \\
10 & 0.7007 \\
50 & 0.7007 \\
100 & 0.7007 \\
500 & 0.7007 \\
1000 & 0.7007 \\
\hline
\end{tabular}

TABLE 6: The superior limit $r_{\text {sup }}$ according to $n_{t}$, which is the order of truncation of the expansion for $f(\kappa)$.

\begin{tabular}{lc}
\hline$n_{t}$ & $r_{\text {sup }}$ \\
\hline 3 & 0.3054 \\
4 & 0.3011 \\
5 & 0.2998 \\
6 & 0.2995 \\
7 & 0.2994 \\
8 & 0.2994 \\
9 & 0.2993 \\
10 & 0.2993 \\
\hline
\end{tabular}

$M_{2}$ : Equilibrium Value Method. If we apply $f(\kappa)=h(\mu)$ in (44), we obtain the result $h(\mu)=0.7599$. However, this value, even though it obeys Ineq. (22), it does not provide a real value for the Gribov parameter $\gamma_{m}^{2}$, according to (45).

$M_{3}$ : Method by Geometric Series. In this case, we will treat the logarithm as a geometric series. For such aim, we employ once again (36) and (37) by treating $r$ as the ratio of a geometric series. In this way, we combine (36), (37), and (44) to find

$$
f(\kappa)=\frac{3}{11} \sum_{n=0}^{n_{t}} r^{n}+\frac{102}{121}\left(\sum_{n=0}^{n_{t}} r^{n}\right)^{2} \ln \left(\sum_{n=0}^{n_{t}} r^{n}\right),
$$

where $n_{t}$ is the truncation order. Now, we must solve the inequality $0<f(\kappa)<1$ to find the range for valid logarithms. Each $n_{t}$ results in an inequality in the form $0<r<r_{\text {sup }}$. To clarify this point, the superior limit $r_{\text {sup }}$ as a function of $n_{t}$ is displayed in Table 6.

The truncation at fourth order works just as good as the 1-loop case because the values for the superior limit for $r$ do not reveal any significant changes. At first sight, we could work with the interval $0<r<0.2993$; however there is another constraint due to the Gribov parameter function $\gamma_{m}^{2}$, according to (46) and (47). The square root in $\gamma_{m}^{2}$ only 
has a real solution if $0.7882<h(\mu)<1$ or, equivalently, $0<r<0.2117$. This interval is actually more restrictive than the 1-loop treatment. Therefore, we are satisfied with the value $r=0.2117$. Hence, using $h(\mu)=0.7883$ in the equation system formed by (44), (45), and (8), we get

$$
\begin{aligned}
& \bar{\Lambda}^{2} \approx 4.593 \times 10^{31} \mathrm{TeV}^{2} \\
& \Lambda^{2} \approx 1.879 \times 10^{32} \mathrm{TeV}^{2}
\end{aligned}
$$

On the other hand, for the other extremes, we choose $r=$ $1.000 \times 10^{-4}$ and the same equation system (see (44), (45), and (8)), we find

$$
\begin{aligned}
& \bar{\Lambda}^{2} \approx 2.269 \times 10^{32} \mathrm{TeV}^{2} \\
& \Lambda^{2} \approx 7.665 \times 10^{31} \mathrm{TeV}^{2}
\end{aligned}
$$

The values (53) match the first ones that we found using $M_{1}$.

4.4. The Logarithmic Elimination Option. A straight and simple manner to simplify the gap equation (45) consists in setting up all logarithms to zero. For this purpose we can set

$$
\frac{\gamma^{2}}{\mu^{2}}=\frac{1}{\sqrt{5}} .
$$

Thus, (45) can easily be solved, providing $N \kappa^{2} / 16 \pi^{2} \approx$ 0.4013 and $\ln \left(\mu^{2} / \bar{\Lambda}^{2}\right) \approx 0.9067$. These results leave us with the following 2-loop values for the energy cut-off and the renormalized cosmological constant:

$$
\begin{aligned}
& \bar{\Lambda}^{2} \approx 1.066 \times 10^{30} \mathrm{TeV}^{2}, \\
& \Lambda^{2} \approx 3.589 \times 10^{31} \mathrm{TeV}^{2} .
\end{aligned}
$$

We remark that, at 1 loop, this strategy is not permitted because the value for the coupling parameter is found to be higher than 1; namely, $N \kappa^{2} / 16 \pi^{2}=1.600$.

\section{Conclusions}

The Gribov mass parameter in our theory is the central point in the development of the induced gravity discussed in [24]. It is responsible for addressing the deformation of the YangMills theory in the infrared regime to a geometrical theory of gravity. In this model, the Gribov parameter and the YangMills coupling constant combine in order to provide the value of Newtonian constant. In the present work we have developed 1- and 2-loop estimates to accommodate reliable values for the prediction of Newtonian constant. Moreover, the renormalization group scale could also be determined. Furthermore, a discussion about the cosmological constant was performed.

Our results show that the experimental value of the Newtonian constant is a solution of the theory and that the renormalization group scale always lie around Planck scale, a good feature for a quantum gravity model candidate. We have also improved the estimates in order to attain the best values for these constants, at 1 and 2 loops.

Concerning the cosmological constant problem, our model provides an inherent gravitational cosmological constant. Following $[48,49]$, it would be nice that the sum of such constant with the one predicted by QFT for the Standard Model vacuum [47] would compensate each other and provide a very small value for the observational cosmological constant. However, although very high, our cosmological constant differs from the QFT prediction by a few orders in magnitude.

Of course, much has to be investigated. For instance, since the emergence of gravity relies on the Gribov parameter and the soft BRST breaking [31, 32, 36], the Gribov-Zwanziger refinement $[34,42,43]$ should also be considered with all the extra mass parameters. These extra masses should also refine the values we have computed in the present work. One interesting feature that should be mentioned of the theory is about the renormalized cosmological constant: first of all, at the quantum level, it is related to the Gribov parameter, so it is a running mass parameter; second, at classical level, its value does not run anymore. Its huge fixed value takes place at the classical sector of the theory. This is very important because it suppresses the quadratic curvature term in (9), at classical level, ensuring the general relativity limit of the theory, at least for a torsionless regime.

\section{Appendix}

\section{A. The Gribov-Zwanziger Action}

We will present here a brief description on the GribovZwanziger scenario. For the details of technicalities and fundamental concepts we refer to [25-30, 71, 72].

Quantization of Yang-Mills theories is a hard work. Initially, the procedure established by Faddeev and Popov [73] succeeded well in the perturbative regime during the process of quantizing the gauge fields. However, the FaddeevPopov method is not accurate at low energies, where the system becomes highly nonperturbative. In essence, a gauge symmetry survives and is manifest at the infrared region. This is the so-called Gribov ambiguities problem. The way to treat such ambiguities, as proposed by Gribov, is to look for a region in the gauge field space without ambiguities and truncate the Faddeev-Popov path integral to such region. Such a region is called fundamental modular region. However the implementation of such region in the Faddeev-Popov path integral is a highly nontrivial problem with no solution so far. Nevertheless, the problem can be partially solved by restricting the path integral to the so-called Gribov region, which is well defined for only a few gauges such as the Landau gauge. At the Landau gauge, the Gribov region can be defined as

$$
\Omega=\left\{Y_{\mu}^{A}, \partial_{\mu} Y_{\mu}^{A}=0, M^{A B}>0\right\}
$$

with $D_{\mu}^{A B}=\delta^{A B} \partial_{\mu}-g f^{A B C} Y^{B} Y^{C}$ and $M^{A B}=-\partial_{\mu} D_{\mu}^{A B}$ is the Faddeev-Popov operator. Following, for instance, [28, 29, 71, 
72], the improved gauge fixed Faddeev-Popov action is given by the Gribov-Zwanziger action:

$$
\begin{aligned}
S_{\mathrm{GZ}}= & S_{Y M}+S_{g f}+\int d^{4} x \gamma^{4} g^{2} f^{A B C} Y_{\mu}^{B} \bar{M}^{A B} f^{D E C} Y_{\mu}^{E} \\
& +\int d^{4} x 4 \gamma^{4}\left(N^{2}-1\right),
\end{aligned}
$$

where $M^{A B}(x) \bar{M}^{B C}(x, y)=\delta^{4}(x-y) \delta^{A C}$. The nonlocal term is known as the horizon function and, together with the gap equation,

$$
\frac{\delta \Gamma}{\delta \gamma^{2}}=0,
$$

ensures that the path integral is inside the Gribov region. In (A.3), $\Gamma$ is the quantum action, determined by

$$
e^{-\Gamma}=\int[d \Psi] e^{-S_{\mathrm{GZ}}}
$$

Remarkably, the nonlocal term can be written in local form with the help of auxiliary fields by means of

$$
e^{-S_{h}}=\int[d \Phi] e^{-S_{\mathrm{loc}}},
$$

with $[d \Phi] \equiv[d \varphi][d \bar{\varphi}][d \omega][d \bar{\omega}]$ and

$$
\begin{aligned}
S_{\mathrm{loc}} & =\int d^{4} x\left[-\bar{\varphi}_{\mu}^{A C} M^{A B} \varphi_{\mu}^{B C}+\bar{\omega}_{\mu}^{A C} M^{A B} \bar{\omega}_{\mu}^{B C}\right. \\
& \left.-\gamma^{2} g f^{A B C} Y_{\mu}^{A}\left(\varphi_{\mu}^{B C}+\varphi_{\mu}^{B C}\right)\right],
\end{aligned}
$$

where the conjugate complex pair $\left(\bar{\varphi}_{\mu}^{A C}, \varphi_{\mu}^{B C}\right)$ are bosonic fields and $\left(\bar{\omega}_{\mu}^{A C}, \omega_{\mu}^{B C}\right)$ are fermionic fields. Hence, the local version of the Gribov-Zwanziger path integral is

$$
\mathscr{Z}=\int[d \Psi] e^{-S_{Y M}-S_{g f}-S_{\mathrm{loc}}-\int d^{4} x 4 \gamma^{4}\left(N^{2}-1\right)},
$$

with $[d \Psi] \equiv[d Y][d \varphi][d \bar{\varphi}][d \omega][d \bar{\omega}][d b][d c][d \bar{c}]$.

The quadratic action (10), as mentioned in Section 3, is obtained from the free part of (A.7). We can straightforwardly observe that the fermionic fields do not contribute to the quadratic action. It is worth mentioning that many contributions from condensates $[34,39]$ also appear in a refined version of (A.7); however, they are not relevant for the purposes of this work.

\section{B. The Choice of the Mass Parameter}

Although we are satisfied with the results obtained in this work, one can argue if another parameter could describe the Newtonian constant and the cosmological constant in a better way. Since we are not considering any condensation effect, the other possibility would be $\lambda$ rather than $\gamma$. Let us reconsider (14) and rewrite the gap equation taking into account $\lambda$ instead of $\gamma$ :

$$
\lambda^{2}=e^{5 / 6} \frac{\mu^{2}}{\left(2 N \kappa^{2}\right)^{1 / 2}} e^{-(4 / 3)\left(16 \pi^{2} / N \kappa^{2}\right)} .
$$

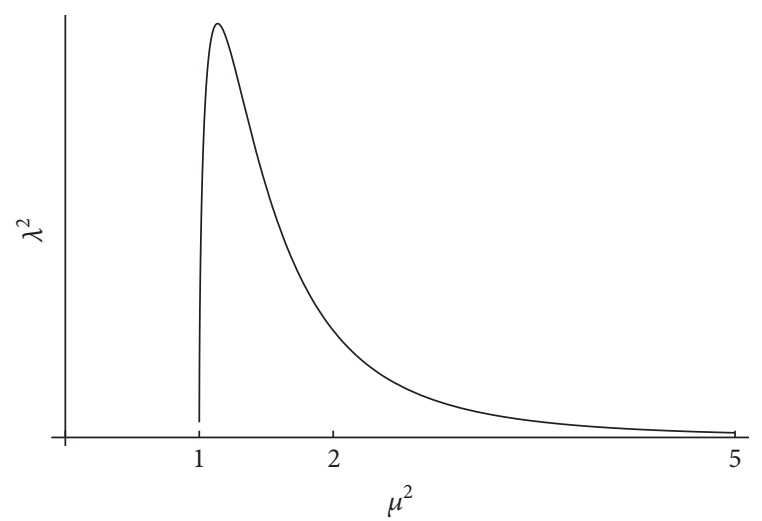

FIGURE 7: Gribov parameter as function of energy scale. The energy is in units of $\bar{\Lambda}$ and the $\lambda^{2}$ parameter is in units of $\xi \bar{\Lambda}^{2}$.

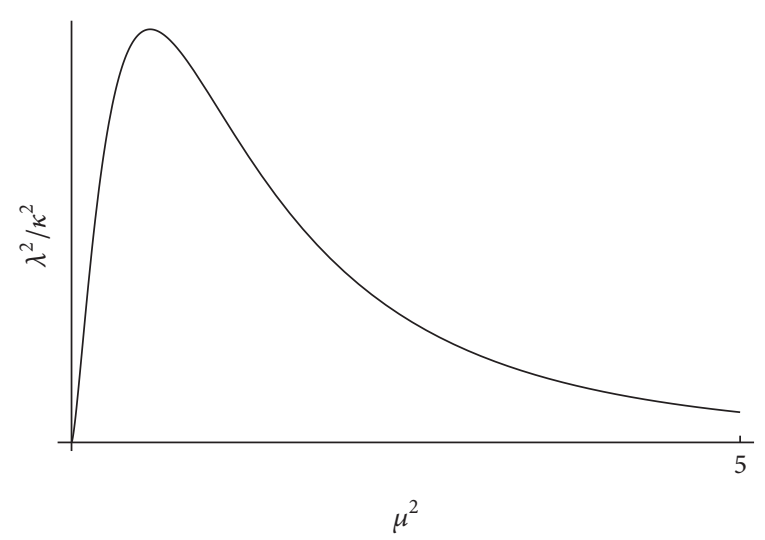

FIGURE 8: The ratio $\lambda^{2} / \kappa^{2} \equiv(4 \pi G)^{-1}$ as function of energy scale $\mu$. The energy scale is in units of $\bar{\Lambda}^{2}$ and $\lambda^{2} / \kappa^{2}$ is in units of $\rho \bar{\Lambda}^{2}$.

Manipulating (18) and (B.1) we get

$$
\lambda^{2}=\xi \bar{\Lambda}^{2}\left(\frac{\mu^{2}}{\bar{\Lambda}^{2}}\right)^{-35 / 9} \ln ^{1 / 2}\left(\frac{\mu^{2}}{\bar{\Lambda}^{2}}\right),
$$

with $\xi=e^{5 / 6} \sqrt{11 / 96 \pi^{2}}$. And (B.2) points again that the smaller the Gribov parameter, the higher the energy scale. We can note in Figure 7 the behaviour of the mass parameter $\lambda^{2}$ when the energy scale rises. Here, we obtain $\lambda^{2}=0$ when $\mu=\bar{\Lambda}$. There is another troublesome here because the mass parameter $\lambda^{2}$ shows itself with the local maximum at $\mu=e^{9 / 140} \bar{\Lambda}$ which indicates-before this point-a decreasing of the mass parameter while the energy scale decreases too. It is completely antagonistic to its physical behaviour at low energy regime where we expect a monotonous rising of the mass parameter while the energy decreases.

From (14), (18), and (19) we get

$$
\frac{\lambda^{2}}{\kappa^{2}}=\rho \bar{\Lambda}^{2}\left(\frac{\mu^{2}}{\bar{\Lambda}^{2}}\right)^{-35 / 9} \ln ^{3 / 2}\left(\frac{\mu^{2}}{\bar{\Lambda}^{2}}\right),
$$

with $\rho=\left(55 / 192 \pi^{3}\right) e^{5 / 6} \sqrt{11 / 6}$. The behaviour of the ratio given by (B.3) can be illustrated in Figure 8. Figure 8 clearly 
shows a nonexistence of a transition because an InonuWigner contraction cannot happen. The vanishing limit of $\lambda^{2} / \kappa^{2}$ only occurs at the origin; hence, no geometric sector would appear. Then, we made the right choice to employ the Gribov parameter $\gamma$ in order to fit with our theory.

\section{Competing Interests}

The authors declare that they have no competing interests.

\section{Acknowledgments}

The Coordenação de Aperfeiçoamento de Pessoal de Nível Superior (CAPES) and the Pró-Reitoria de Pesquisa, PósGraduação e Inovação (PROPPI-UFF) are acknowledged.

\section{Endnotes}

1. Even though most of the material in this section can be found in previous articles [24, 45, 46], some new aspects are not fully discussed there.

2. Although we did not specify the gauge fixing constraint, the Gribov problem is a pathological issue plaguing all covariant gauges [26]. Nevertheless, we can anticipate for the reader that we will employ the Landau gauge fixing in this entire work.

3. The renormalized cosmological constant has associated with the ansatz of the theory; that is, $\Lambda_{\mathrm{obs}}^{2}=\Lambda_{\mathrm{qft}}^{2}+$ $\Lambda^{2}$.

4. From now on, for the sake of simplicity, we use tensorial notation with Greek indices indicating spacetime coordinates and Latin indices to gauge group.

5. See Appendix A and the references therein for details.

6. At this level, we are not considering the refined GribovZwanziger action [30].

7. Strictly speaking, (11) does not define the effective action since there are no source terms. In fact, $\Gamma^{(1)}$ is rather a field-independent shift of the actual 1-loop effective action. This is sufficient for our purposes but does not constitute the full effective action. Nevertheless, since there is no formal term for it, we will call it simply as effective action in this work. We expect no confusion for the reader.

8. We notice that $r<1$ due to Ineq. (22).

\section{References}

[1] A. Ashtekar and J. Lewandowski, "Background independent quantum gravity: a status report," Classical and Quantum Gravity, vol. 21, no. 15, article R53, 2004.

[2] C. Rovelli and F. Vidotto, Covariant Loop Quantum Gravity: An Elementary Introduction to Quantum Gravity and Spinfoam Theory, Cambridge University Press, 2014.
[3] M. Asorey, J. L. López, and I. L. Shapiro, "Some remarks on high derivative quantum gravity," International Journal of Modern Physics A: Particles and Fields. Gravitation. Cosmology, vol. 12, no. 32, pp. 5711-5734, 1997.

[4] I. L. Buchbinder, S. D. Odintsov, and I. L. Shapiro, Effective Action in Quantum Gravity, IOP, Bristol, UK, 1992.

[5] J. Henson, "The causal set approach to quantum gravity" in Approaches to Quantum Gravity-Towards a New Understanding of Space and Time, D. Oriti, Ed., pp. 393-413, Cambridge University Press, New York, NY, USA, 2006.

[6] J. Ambjørn, A. Görlich, J. Jurkiewicz, and R. Loll, "Nonperturbative quantum gravity," Physics Reports, vol. 519, no. 4-5, pp. 127-210, 2012.

[7] E. Witten, "Anti de Sitter space and holography," Advances in Theoretical and Mathematical Physics, vol. 2, no. 2, pp. 253-291, 1998.

[8] N. Seiberg and E. Witten, "String theory and noncommutative geometry," Journal of High Energy Physics, vol. 1999, no. 9, article 032, 1999.

[9] S. W. Hawking and W. Israel, General Relativity: An Einstein Centenary Survey, Cambridge University Press, Cambridge, UK, 1979.

[10] M. Reuter and F. Saueressig, "Quantum Einstein gravity," New Journal of Physics, vol. 14, Article ID 055022, 2012.

[11] C. Barceló, M. Visser, and S. Liberati, "Einstein gravity as an emergent phenomenon?" International Journal of Modern Physics D: Gravitation, Astrophysics, Cosmology, vol. 10, no. 6, pp. 799-806, 2001.

[12] P. Hořava, "Quantum gravity at a Lifshitz point," Physical Review $D$, vol. 79, no. 8, Article ID 084008, 15 pages, 2009.

[13] S. Nojiri and S. D. Odintsov, "Covariant renormalizable gravity and its FRW cosmology," Physical Review D, vol. 81, no. 4, Article ID 043001, 2010.

[14] G. Cognola, R. Myrzakulov, L. Sebastiani, S. Vagnozzi, and S. Zerbini, "Covariant renormalizable gravity model as a mimetic Horndeski model: cosmological solutions and perturbations," https://arxiv.org/abs/1601.00102.

[15] J. C. Baez, "An introduction to spin foam models of BF theory and quantum gravity," in Geometry and Quantum Physics: Proceeding of the 38. Internationale Universitätswochen für Kernund Teilchenphysik, Schladming, Austria, January 9-16, 1999, vol. 543 of Lecture Notes in Physics, pp. 25-93, Springer, Berlin, Germany, 2000.

[16] C. Itzykson and J. B. Zuber, Quantum Field Theory, International Series In Pure and Applied Physics, McGraw-Hill Education, New York, NY, USA, 1980.

[17] T. P. Cheng and L. F. Li, Gauge Theory of Elementary Particle Physics, Clarendon, Oxford Science Publications, Oxford, UK, 1984.

[18] K. A. Olive, K. Agashe, C. Amsler et al., "Review of particle physics," Chinese Physics C, vol. 38, no. 9, Article ID 090001, 2014.

[19] R. Utiyama, "Invariant theoretical interpretation of interaction," Physical Review, vol. 101, no. 5, p. 1597, 1956.

[20] T. W. B. Kibble, "Lorentz invariance and the gravitational field," Journal of Mathematical Physics, vol. 2, no. 2, pp. 212-221, 1961.

[21] D. W. Sciama, "The physical structure of general relativity," Reviews of Modern Physics, vol. 36, no. 1, pp. 463-469, 1964, Erratum in: Reviews of Modern Physics, vol. 36, no. 4, p. 1103, 1964. 
[22] A. Trautman, "Fiber bundles, gauge fields, and gravitation," in General Relativity and Gravitation, A. Held, Ed., vol. 1, pp. 287308, Plenum Press, New York, NY, USA, 1980.

[23] A. Mardones and J. Zanelli, "Lovelock-Cartan theory of gravity," Classical and Quantum Gravity, vol. 8, no. 8, pp. 1545-1558, 1991.

[24] R. F. Sobreiro, A. A. Tomaz, and V. J. V. Otoya, "de Sitter gauge theories and induced gravities," The European Physical Journal C, vol. 72, article 1991, 2012.

[25] V. N. Gribov, "Quantization of non-Abelian gauge theories," Nuclear Physics B, vol. 139, no. 1-2, pp. 1-19, 1978.

[26] I. M. Singer, "Some remarks on the Gribov ambiguity," Communications in Mathematical Physics, vol. 60, no. 1, pp. 7-12, 1978.

[27] D. Zwanziger, "Action from the Gribov horizon," Nuclear Physics B, vol. 321, no. 3, pp. 591-604, 1989.

[28] D. Zwanziger, "Local and renormalizable action from the Gribov horizon," Nuclear Physics B, vol. 323, no. 3, pp. 513-544, 1989.

[29] R. F. Sobreiro and S. P. Sorella, "Introduction to the Gribov ambiguities in Euclidean Yang-Mills theories," in Proceedings of the Lectures given by SPS at the 13th Jorge Andre Swieca Summer School on Particles and Fields, Campos de Jordão, Brazil, January 2005.

[30] D. Dudal, R. F. Sobreiro, S. P. Sorella, and H. Verschelde, "Gribov parameter and the dimension two gluon condensate in Euclidean Yang-Mills theories in the Landau gauge," Physical Review D, vol. 72, no. 1, Article ID 014016, 2005.

[31] L. Baulieu and S. P. Sorella, "Soft breaking of BRST invariance for introducing non-perturbative infrared effects in a local and renormalizable way," Physics Letters B, vol. 671, no. 4-5, pp. 481485, 2009.

[32] L. Baulieu, M. A. L. Capri, A. J. Gómez, V. E. R. Lemes, R. F. Sobreiro, and S. P. Sorella, "Renormalizability of a quark-gluon model with soft BRST breaking in the infrared region," The European Physical Journal C, vol. 66, no. 3, pp. 451-464, 2010.

[33] D. Dudal, M. S. Guimaraes, and S. P. Sorella, "Glueball masses from an infrared moment problem," Physical Review Letters, vol. 106, no. 6, Article ID 062003, 2011.

[34] D. Dudal, S. P. Sorella, and N. Vandersickel, "Dynamical origin of the refinement of the Gribov-Zwanziger theory," Physical Review D, vol. 84, no. 6, Article ID 065039, 2011.

[35] M. A. L. Capri, D. Dudal, M. S. Guimaraes, L. F. Palhares, and S. P. Sorella, "Physical spectrum from confined excitations in a Yang-Mills-inspired toy model," International Journal of Modern Physics A, vol. 28, no. 10, Article ID 1350034, 2013.

[36] D. Dudal and S. P. Sorella, "Gribov horizon and spontaneous BRST symmetry breaking," Physical Review D, vol. 86, no. 4, Article ID 045005, 2012.

[37] A. D. Pereira and R. F. Sobreiro, "On the elimination of infinitesimal Gribov ambiguities in non-Abelian gauge theories," The European Physical Journal C, vol. 73, article 2584, 2013.

[38] D. Dudal, M. S. Guimaraes, L. F. Palhares, and S. P. Sorella, "Confinement and dynamical chiral symmetry breaking in a non-perturbative renormalizable quark model," Annals of Physics, vol. 365, pp. 155-179, 2016.

[39] M. A. L. Capri, D. Dudal, M. S. Guimaraes, I. F. Justo, L. F. Palhares, and S. P. Sorella, "Spontaneous breaking of the BRST symmetry in the presence of the Gribov horizon: renormalizability," Annals of Physics, vol. 339, pp. 344-357, 2013.

[40] M. A. L. Capri, A. D. Pereira, R. F. Sobreiro, and S. P. Sorella, "Non-perturbative treatment of the linear covariant gauges by taking into account the Gribov copies," The European Physical Journal C, vol. 75, no. 10, article 479, 2015.
[41] M. A. L. Capri, D. Fiorentini, M. S. Guimaraes et al., "Exact nilpotent nonperturbative BRST symmetry for the GribovZwanziger action in the linear covariant gauge," Physical Review D, vol. 92, no. 4, Article ID 045039, 2015.

[42] M. A. L. Capri, D. Fiorentini, M. S. Guimaraes et al., "More on the nonperturbative Gribov-Zwanziger quantization of linear covariant gauges," Physical Review D, vol. 93, no. 6, Article ID 065019, 13 pages, 2016.

[43] M. A. L. Capri, D. Dudal, D. Fiorentini et al., "Local and BRST-invariant Yang-Mills theory within the Gribov horizon," Physical Review D, vol. 94, no. 2, Article ID 025035, 2016.

[44] E. Inonu and E. P. Wigner, "On the contraction of groups and their representations," Proceedings of the National Academy of Sciences of the United States of America, vol. 39, no. 6, pp. 510524, 1953.

[45] R. F. Sobreiro, A. A. Tomaz, and V. J. Vasquez Otoya, "Induced gravity from gauge theories," Journal of Physics: Conference Series, vol. 453, Article ID 012014, 2013.

[46] R. F. Sobreiro, A. A. Tomaz, and V. J. V. Otoya, "Gauge theories and gravity," http://arxiv.org/abs/1210.8446.

[47] S. Weinberg, "The cosmological constant problem," Reviews of Modern Physics, vol. 61, no. 1, pp. 1-23, 1989.

[48] I. L. Shapiro and J. Solà, "Cosmological constant problems and the renormalization group," Journal of Physics A: Mathematical and Theoretical, vol. 40, no. 25, pp. 6583-6593, 2007.

[49] I. L. Shapiro and J. Solà, "On the possible running of the cosmological 'constant,' Physics Letters B, vol. 682, no. 1, pp. 105-113, 2009.

[50] T. S. Assimos, A. D. Pereira, T. R. S. Santos, R. F. Sobreiro, A. A. Tomaz, and V. J. V. Otoya, "Dark gravity from a renormalizable gauge theory," http://arxiv.org/abs/1305.1468.

[51] O. Piguet and S. P. Sorella, Algebraic Renormalization: Perturbative Renormalization, Symmetries and Anomalies, vol. 28 of Lecture Notes in Physics Monographs, Springer, Berlin, Germany, 1995.

[52] D. J. Gross and F. Wilczek, "Ultraviolet behavior of non-Abelian Gauge theories," Physical Review Letters, vol. 30, no. 26, p. 1343, 1973.

[53] H. D. Politzer, "Reliable Perturbative Results for Strong Interactions?" Physical Review Letters, vol. 30, no. 26, pp. 1346-1349, 1973.

[54] D. Zwanziger, "Renormalizability of the critical limit of lattice gauge theory by BRS invariance," Nuclear Physics B, vol. 399, no. 2-3, pp. 477-513, 1993.

[55] N. Maggiore and M. Schaden, "Landau gauge within the Gribov horizon," Physical Review D, vol. 50, no. 10, pp. 6616-6625, 1994.

[56] A. D. Pereira, R. F. Sobreiro, and S. P. Sorella, "Non-perturbative BRST quantization of Euclidean Yang-Mills theories in CurciFerrari gauges," https://arxiv.org/abs/1605.09747.

[57] M. A. L. Capri, D. Fiorentini, A. D. Pereira, R. F. Sobreiro, S. P. Sorella, and R. C. Terin, "Aspects of the refined GribovZwanziger action in linear covariant gauges," https://arxiv.org/ abs/1607.07912.

[58] J. C. R. Bloch, A. Cucchieri, K. Langfeld, and T. Mendes, "Propagators and running coupling from SU(2) lattice gauge theory," Nuclear Physics B, vol. 687, no. 1-2, pp. 76-100, 2004.

[59] A. Cucchieri and T. Mendes, "Propagators, running coupling and condensates in lattice QCD," Brazilian Journal of Physics, vol. 37, no. 2, pp. 484-493, 2007.

[60] A. Cucchieri and T. Mendes, "Landau-gauge propagators in Yang-Mills theories at $\beta=0$ : massive solution versus conformal 
scaling," Physical Review D, vol. 81, no. 1, Article ID 016005, 17 pages, 2010.

[61] A. Cucchieri, D. Dudal, and N. Vandersickel, "No-pole condition in Landau gauge: properties of the Gribov ghost form factor and a constraint on the $2 d$ gluon propagator," Physical Review D, vol. 85, no. 8, Article ID 085025, 1912.

[62] A. Cucchieri, D. Dudal, T. Mendes, and N. Vandersickel, "BRST-symmetry breaking and Bose-ghost propagator in lattice minimal Landau gauge," Physical Review D, vol. 90, no. 5, Article ID 051501, 2014.

[63] A. Cucchieri, D. Dudal, T. Mendes, N. Vandersickel, and D. Phys. Rev, “doi:10.1103/PhysRevD.93. [”" Phys. Rev. D, vol. 9, Article ID 094513, 2016, arXiv:1602.01646.

[64] P. Nogueira, “Automatic feynman graph generation," Journal of Computational Physics, vol. 105, no. 2, pp. 279-289, 1993.

[65] F. R. Ford and J. A. Gracey, "Two-loop $\overline{M S}$ Gribov mass gap equation with massive quarks," Journal of Physics A: Mathematical and Theoretical, vol. 42, no. 32, Article ID 325402, 2009, Erratum-ibid, vol. 43, no. 22, Article ID 229802, 2010.

[66] J. Kuipers, T. Ueda, J. A. M. Vermaseren, and J. Vollinga, "FORM version 4.0," Computer Physics Communications, vol. 184, no. 5, pp. 1453-1467, 2013.

[67] J. Kuipers, T. Ueda, and J. A. M. Vermaseren, "Code optimization in FORM," Computer Physics Communications, vol. 189, pp. 1-19, 2015.

[68] T. Ueda and J. Vermaseren, "Recent developments on FORM," Journal of Physics: Conference Series, vol. 523, Article ID 012047, 2014.

[69] M. Steinhauser, T. Ueda, and J. A. M. Vermaseren, "Parallel versions of FORM and more," Nuclear and Particle Physics Proceedings, vol. 261-262, pp. 45-57, 2015.

[70] J. C. Collins, Renormalization: An Introduction to Renormalization, The Renormalization Group, and the Operator Product Expansion, Cambridge University Press, Cambridge, UK, 1984.

[71] S. P. Sorella, "Gribov horizon and BRST symmetry: a few remarks," Physical Review D, vol. 80, no. 2, Article ID 025013, 12 pages, 2009.

[72] D. Dudal, N. Vandersickel, H. Verschelde, and S. P. Sorella, "Aspects of the Gribov-Zwanziger framework," https://arxiv .org/abs/0911.0082.

[73] L. D. Faddeev and V. N. Popov, "Feynman diagrams for the Yang-Mills field," Physics Letters B, vol. 25, no. 1, pp. 29-30, 1967. 

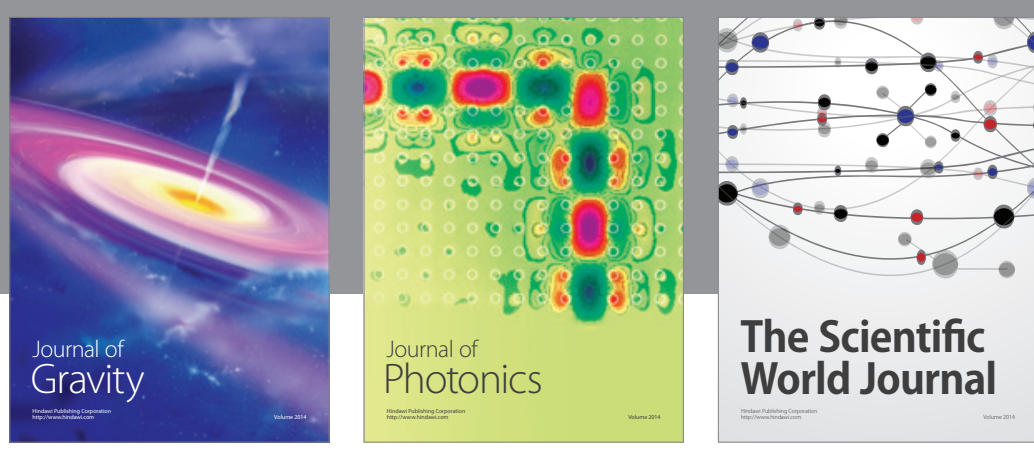

The Scientific World Journal
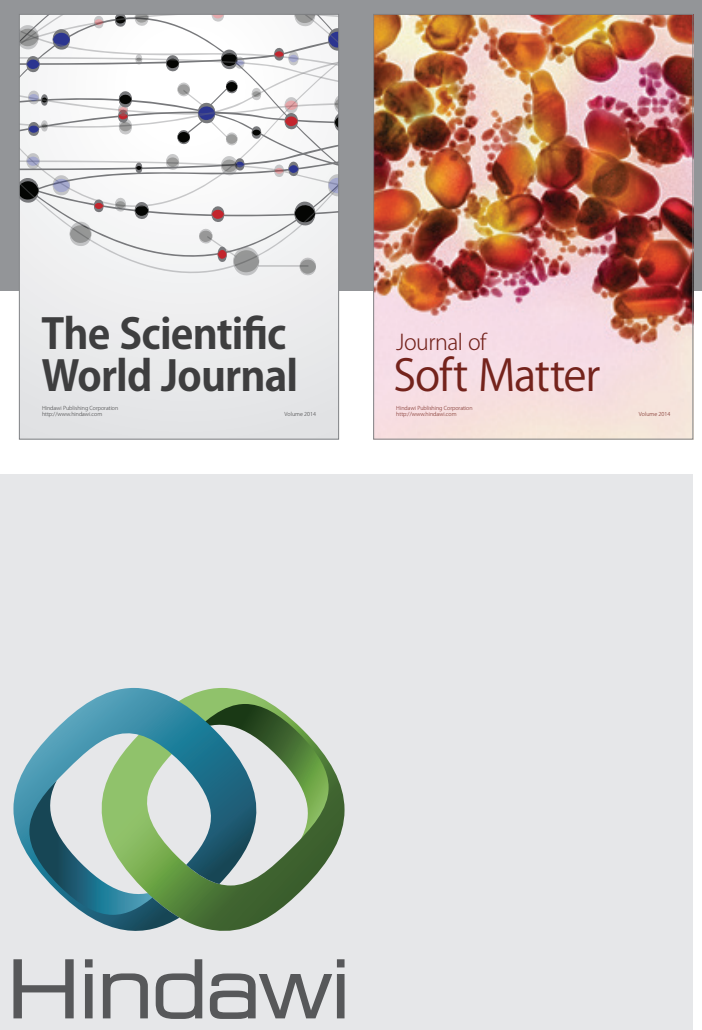

Submit your manuscripts at

http://www.hindawi.com

nternational Journal of

Statistical Mechanics
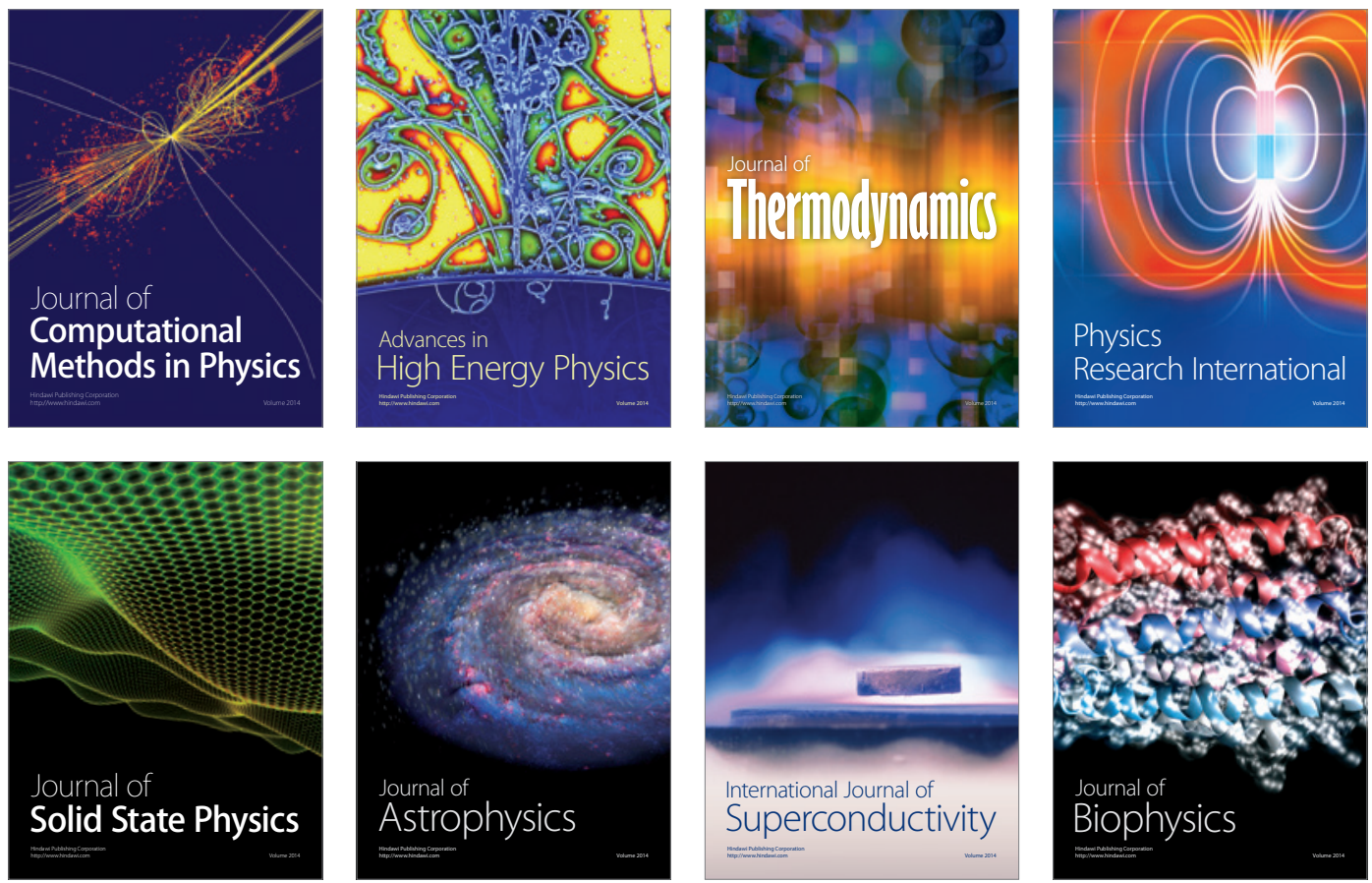
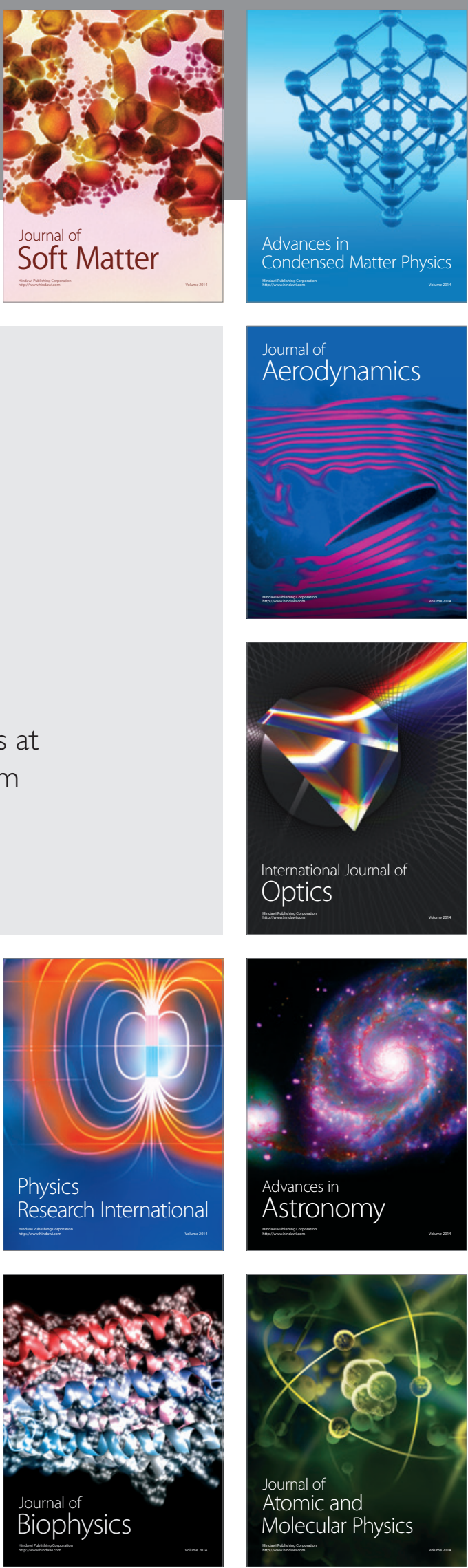